\title{
Prognostic factors for adverse outcomes in patients with COVID- 19: a field-wide systematic review and meta-analysis
}

\author{
Vanesa Bellou $\mathbb{1}^{1}$, loanna Tzoulaki $\mathbb{1}^{1,2,3}$, Maarten van Smeden $\mathbb{1}^{4}$, Karel G.M. Moons $\mathrm{s}^{4,5}$, \\ Evangelos Evangelou (i) ${ }^{1,2,3}$ and Lazaros Belbasis (i) ${ }^{1}$
}

${ }^{1}$ Dept of Hygiene and Epidemiology, University of Ioannina Medical School, Ioannina, Greece. ${ }^{2}$ Dept of Epidemiology and Biostatistics, School of Public Health, Imperial College London, London, UK. ${ }^{3}$ Institute of Biosciences, University Research Center of Ioannina, Ioannina, Greece. ${ }^{4}$ Julius Center for Health Sciences and Primary Care, University Medical Centre Utrecht, Utrecht University, Utrecht, The Netherlands. ${ }^{5}$ Cochrane Netherlands, University Medical Centre Utrecht, Utrecht University, Utrecht, The Netherlands.

Corresponding author: Lazaros Belbasis (lbelbasis@cc.uoi.gr)

Shareable abstract (@ERSpublications)

ACE inhibitors, sleep apnoea, pharyngalgia, VTE history, sex, coronary heart disease, cancer, liver disease, COPD, dementia, immunosuppressive drugs, peripheral arterial disease, rheumatological disease and smoking predict outcomes of COVID-19 patients https://bit.ly/3plaANC

Cite this article as: Bellou V, Tzoulaki I, van Smeden M, et al. Prognostic factors for adverse outcomes in patients with COVID-19: a field-wide systematic review and meta-analysis. Eur Respir J 2022; 59: 2002964 [DOI: 10.1183/13993003.02964-2020].

Copyright $\odot$ The authors 2022

This version is distributed under the terms of the Creative Commons Attribution Non-Commercial Licence 4.0. For commercial reproduction rights and permissions contact permissions@ersnet.org

Received: 29 July 2020 Accepted: 4 June 2021

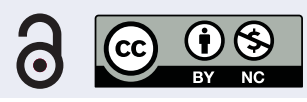

\section{Abstract}

Introduction The individual prognostic factors for coronavirus disease 2019 (COVID-19) are unclear. For this reason, we aimed to present a state-of-the-art systematic review and meta-analysis on the prognostic factors for adverse outcomes in COVID-19 patients.

Methods We systematically reviewed PubMed from 1 January 2020 to 26 July 2020 to identify nonoverlapping studies examining the association of any prognostic factor with any adverse outcome in patients with COVID-19. Random-effects meta-analysis was performed, and between-study heterogeneity was quantified using $\mathrm{I}^{2}$ statistic. Presence of small-study effects was assessed by applying the Egger's regression test.

Results We identified 428 eligible articles, which were used in a total of 263 meta-analyses examining the association of 91 unique prognostic factors with 11 outcomes. Angiotensin-converting enzyme inhibitors, obstructive sleep apnoea, pharyngalgia, history of venous thromboembolism, sex, coronary heart disease, cancer, chronic liver disease, COPD, dementia, any immunosuppressive medication, peripheral arterial disease, rheumatological disease and smoking were associated with at least one outcome and had $>1000$ events, $\mathrm{p}<0.005, \mathrm{I}^{2}<50 \%$, 95\% prediction interval excluding the null value, and absence of small-study effects in the respective meta-analysis. The risk of bias assessment using the Quality in Prognosis Studies tool indicated high risk of bias in 302 out of 428 articles for study participation, 389 articles for adjustment for other prognostic factors and 396 articles for statistical analysis and reporting.

Conclusions Our findings could be used for prognostic model building and guide patient selection for randomised clinical trials.

\section{Introduction}

In December 2019, a cluster of pneumonia cases was reported in Wuhan, China, and subsequent epidemiological tracking identified a novel coronavirus (severe acute respiratory syndrome coronavirus 2 (SARS-CoV-2)) as the cause [1]. SARS-CoV-2 has spread across all continents since then and caused a public health crisis [2]. As of February 2021, there have been $>100$ million confirmed cases with coronavirus disease 2019 (COVID-19) and >2 million deaths according to the World Health Organization [3].

The ongoing public health emergency necessitates the discovery of reliable prognostic factors to guide clinical decision-making and treatment plans tailored to patient characteristics. In addition, these prognostic factors could improve the design and analysis of future clinical trials and suggest novel insights into the molecular pathways of the disease $[4,5]$. 
There is a growing body of literature on COVID-19 patients, examining prognostic features of the disease. However, clinical decision-making and research guidance is often based on narrative reviews or low-quality studies [6]. There is as yet no published effort to critically and systematically summarise the epidemiological evidence on the entire prognostic factor landscape for multiple adverse outcomes in COVID-19 patients. Our study aims to fill this gap by conducting the first comprehensive systematic review, critical appraisal and (in case of sufficient data) meta-analysis of all prognostic factors in patients with COVID-19 by applying state-of-the-art approaches.

\section{Methods}

We followed the Meta-analysis of Observational Studies in Epidemiology guideline and the latest guidance of the Prognosis Methods Group of the Cochrane Collaboration to design and report our meta-analysis [7, 8]. We designed the research question and data extraction of this systematic review according to the modified checklist for Critical Appraisal and Data Extraction for Systematic Reviews of Prognostic Factor studies (CHARMS-PF) [5, 9]. A protocol for this systematic review was published on Open Science Foundation (https://osf.io/382wj).

\section{Literature search}

We systematically searched PubMed from 1 January 2020 to 26 July 2020 to identify all studies examining any prognostic factor of any adverse outcome in patients with COVID-19. Our search algorithm was based on LitCovid [10, 11] and was as follows: “coronavirus”[All Fields] OR “SARS-CoV-2”[All Fields] OR “cov”[All Fields] OR “2019-SARS-CoV-2”[All Fields] OR “COVID-19”[All Fields]. The literature search was performed by two independent researchers (VB, LB). Additionally, we performed a reference screening of the eligible articles to identify additional potentially eligible articles.

\section{Eligibility criteria}

We considered as eligible observational studies or clinical trials that included patients with SARS-CoV-2 infection (defined by real-time PCR testing or standardised clinical/radiological criteria) and examined the effect of any prognostic factor on any outcome. We considered as eligible any outcome that was relevant to patients if its definition and measurement was standardised or explicitly defined. We excluded outcomes measured at the time of COVID-19 diagnosis or hospital admission, and prognostic factors measured after COVID-19 diagnosis or hospital admission, to ensure temporality between prognostic factor and outcome.

Prognostic factor was defined as "any measure that, among people with a given health condition, is associated with a subsequent clinical outcome” [12]. We considered all types of prognostic factors including demographic and anthropometric individual characteristics, biomarkers, symptoms, clinical signs, medical history and comorbid diseases, medications, and findings in chest imaging. Table 1 shows a detailed description of the PICOTS (Population, Index prognostic factor, Comparator prognostic factor, Outcome, Timing, Setting) system [5, 9, 13].

\section{Data extraction}

Data extraction was performed independently by two researchers (VB, LB) following the CHARMS checklist [5, 9]. From each article, we extracted the first author, the year and the journal of publication, the geographic region and the hospital involved, the recruitment period, the examined prognostic factors and

TABLE 1 Key items for framing aim, search strategy, and study inclusion and exclusion criteria for systematic review, following PICOTS (Population, Index prognostic factor, Comparator prognostic factor, Outcome, Timing, Setting) system

\section{Definition}

\section{Population}

Index prognostic factors

Comparator prognostic factors

Outcomes

Timing

Setting
Patients diagnosed with COVID-19

Any variable that was measured before hospital admission or diagnosis of COVID-19 and was examined for an association with any adverse event

Not applicable

Any clearly defined adverse event in patients with COVID-19

Prognostic factors measured before diagnosis of COVID-19 or hospital admission, and predicting adverse outcomes at any time horizon

Patients visiting ambulatory healthcare facilities, patients admitted to hospital, or patients visiting emergency department

COVID-19: coronavirus disease 2019. 
the examined outcomes and their definitions. For each prognostic factor, we extracted the reported measure of association (i.e. odds ratio, risk ratio or hazard ratio) and the level of comparison. We extracted both univariable and multivariable prognostic factor's effect estimates, whenever available [5, 8].

Risk-of-bias assessment

The presence of bias in the eligible studies was assessed independently by two researchers (VB, LB) using the Quality in Prognosis Studies (QUIPS) tool. QUIPS assesses six domains: participation, attrition, prognostic factor measurement, adjustment for other prognostic factors, outcome measurement, and statistical analysis and reporting $[5,14]$. In each domain, risk of bias was assessed as low, moderate or high based on a pre-specified set of questions.

\section{Statistical analysis}

In the absence of a reported prognostic effect size, we calculated the univariable odds ratio and its standard error from the reported $2 \times 2$ contingency table. When zero counts occurred in a cell of the contingency table, we applied the Haldane-Anscombe correction [15].

For associations examined in at least five studies, we estimated the summary effect estimate and its 95\% confidence interval applying the DerSimonian and Laird random-effects model, because methodological heterogeneity was expected between the eligible studies [16, 17]. To minimise the effect of different outcome definitions on between-study heterogeneity, we combined only studies using the same outcome definition. A statistically significant effect was claimed at $\mathrm{p}<0.05$. Additionally, between-study heterogeneity was quantified by the $\mathrm{I}^{2}$ statistic $[18,19]$. Values $>50 \%$ and $>75 \%$ were judged as large and very large heterogeneity, respectively.

We estimated the 95\% prediction interval, which further accounts for the uncertainty for the effect that would be expected in a new study addressing the same association [16]. We assessed whether there was evidence for small-study effects using Egger's regression asymmetry test [20]. The presence of small-study effects was based on a statistically significant Egger's test at $p<0.10$ combined with a more conservative effect in the largest study of the meta-analysis compared to the effect in the random-effects meta-analysis.

We performed three sensitivity analyses to explore potential sources of between-study heterogeneity by excluding 1) studies presenting hazard ratios, 2) studies with a sample <100 COVID-19 patients and 3) studies including only individuals with specific comorbidities. Then, we estimated the Spearman correlation coefficient to examine the correlation of effect estimate, $\mathrm{I}^{2}$ statistic and p-value between the main analysis and the sensitivity analyses.

In addition, we examined which associations presented a highly significant effect at $\mathrm{p}<0.005$, absence of large or very large between-study heterogeneity (i.e. $\mathrm{I}^{2}<50 \%$ ), 95\% prediction interval excluding the null value, >1000 events and absence of small-study effects. The rationale for the use of a strict p-value threshold is based on current recommendations to avoid false-positive findings [21-23].

Statistical analysis was performed on R Statistical Software (version 3.6.3; Foundation for Statistical Computing, Vienna, Austria) and the packages "metaphor" and "metareg” were used for all analyses. Additional details on the eligibility criteria, the data extraction process and the risk of bias assessment are presented in the supplementary material.

\section{Results}

Description of eligible articles

We screened 36661 articles, and we identified 428 eligible articles that were published between 1 January 2020 and 26 July 2020 and were included in a meta-analysis (figure 1). The majority of the eligible studies were from China $(n=106)$, USA $(n=98)$, Italy $(n=65)$, Spain $(n=34)$, France $(n=26)$ and UK $(n=20)$.

\section{Risk-of-bias assessment}

424 (99\%) out of 428 eligible articles that participated in at least one meta-analysis were graded as having high risk of bias in at least one domain (figure 2). To specify on domains, high risk of bias was present in 302 (71\%) articles for study participation, one (0.2\%) article for study attrition, 255 (60\%) articles for prognostic factor measurement, 19 (4\%) articles for outcome measurement and 396 (93\%) articles for statistical analysis and reporting. 389 (91\%) articles did not present a prognostic factor's effect estimate that was adjusted for other prognostic factors. Detailed assessment of the eligible articles per domain is presented in supplementary table S1. 


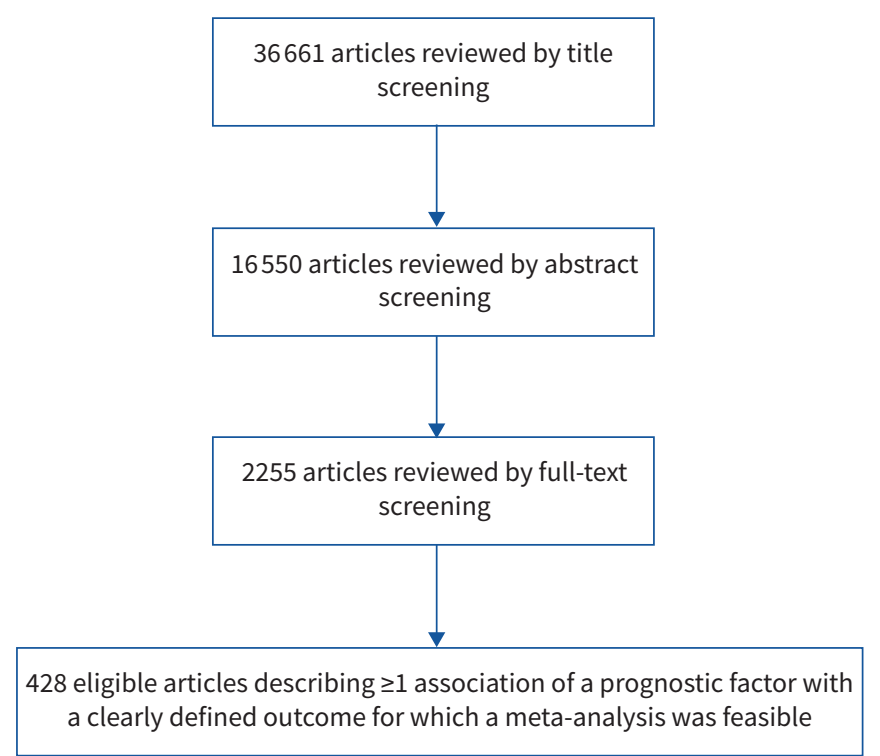

FIGURE 1 Flow chart of literature search for individual prognostic factors in patients with coronavirus disease 2019.

\section{Description of clinical outcomes and prognostic factors}

We performed 263 meta-analyses focused on risk for mortality (89 meta-analyses), hospital admission (50 meta-analyses), intensive care unit (ICU) admission (48 meta-analyses), two composite outcomes (24 meta-analyses), invasive mechanical ventilation (23 meta-analyses), acute kidney injury (nine meta-analyses), venous thromboembolism (six meta-analyses), pulmonary embolism (five meta-analyses), acute respiratory distress syndrome (five meta-analyses) and deep venous thrombosis (four meta-analyses).

Overall, we evaluated 91 unique prognostic factors, which were categorised into seven categories: biomarkers (16 meta-analyses, 11 unique factors), comorbidities (120 meta-analyses, 30 unique factors), imaging markers (seven meta-analyses, four unique factors), demographic characteristics (25 meta-analyses, three unique factors), environmental factors (eight meta-analyses, two unique factors), medications (28 meta-analyses, 12 unique factors), and symptoms or clinical signs (59 meta-analyses, 29 unique factors).

\section{Findings from meta-analyses}

The median (interquartile range (IQR)) number of studies per meta-analysis was 9 (6-14); the median number of events per meta-analysis was 1195 (514-2937); and the median number of COVID-19 participants per meta-analysis was 5131 (2394-13395). 157 (60\%) out of 263 meta-analyses included $>1000$ events. 149 (57\%) out of 263 meta-analyses presented a statistically significant effect at $\mathrm{p}<0.05$, whereas 119 of them had a p-value $<0.005$.

$137(52 \%)$ out of 263 meta-analyses presented large or very large between-study heterogeneity $\left(I^{2} \geqslant 50 \%\right)$. $48(18 \%)$ out of 263 meta-analyses presented a 95\% prediction interval that excluded the null value. In addition, 18 (7\%) out of 263 meta-analyses presented evidence for small-study effects. The results of the meta-analyses for the 263 associations are available in supplementary table S2.

Out of the 149 statistically significant meta-analyses at $\mathrm{p}<0.05,16$ meta-analyses included $>1000$ events, had $\mathrm{p}<0.005$ in the random-effects model, $\mathrm{I}^{2}<50 \%, 95 \%$ prediction interval excluding the null value and absence of small-study effects. These meta-analyses examined the effect of obstructive sleep apnoea (OR 2.11, 95\% CI 1.54-2.89) and history of venous thromboembolism (OR 2.35, 95\% CI 1.75-3.14) on risk of hospitalisation; the effect of female sex (OR 0.53, 95\% CI 0.46-0.59) on risk of ICU admission; the effect of coronary heart disease (OR 1.69, 95\% CI 1.43-1.99) on risk of acute kidney injury; the effect of cancer (OR 1.57, 95\% CI 1.25-1.98) and female sex (OR 0.62, 95\% CI 0.55-0.71) on risk of invasive mechanical ventilation; and the effect of angiotensin-converting enzyme inhibitors (ACEi) (OR 1.38, 95\% CI 1.20-1.59), cancer (OR 2.14, 95\% CI 1.86-2.45), chronic liver disease (OR 1.46, 95\% CI 1.24-1.72), COPD (OR 2.19, 95\% CI 1.88-2.55), dementia (OR 3.12, 95\% CI 2.50-3.90), any immunosuppressive medication (OR 1.22, 


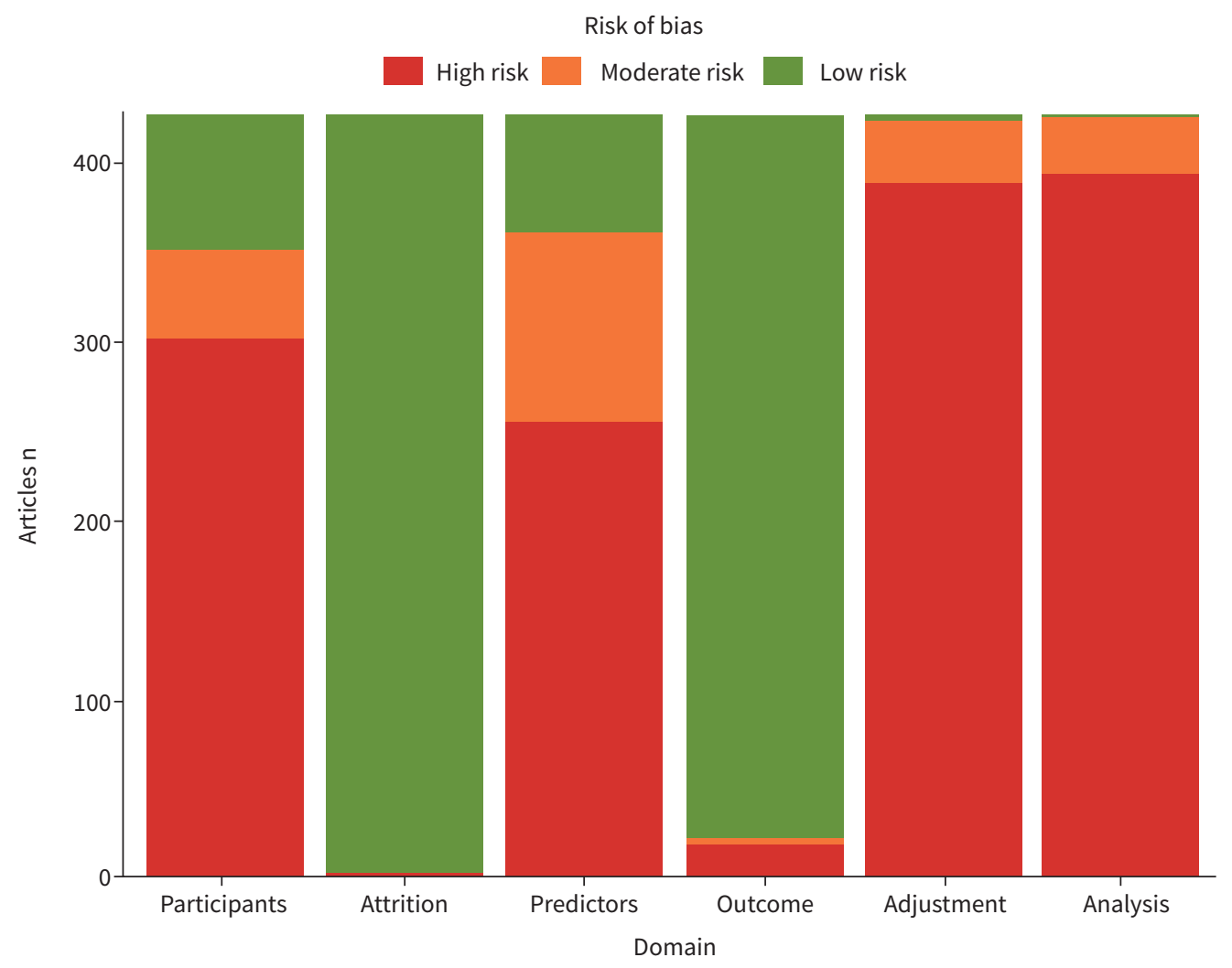

FIGURE 2 Risk-of-bias assessment (using Quality in Prognosis Studies tool) based on six domains across 428 eligible articles for adverse outcomes in patients with coronavirus disease 2019.

95\% CI 1.12-1.34), peripheral arterial disease (OR 2.07, 95\% CI 1.55-2.75), pharyngalgia (OR 0.58, 95\% CI 0.46-0.71), rheumatological disease (OR 1.41, 95\% CI 1.27-1.57) and smoking (OR 1.50, 95\% CI 1.351.66) on risk of mortality. 13 additional meta-analyses fulfilled the aforementioned characteristics but had a 95\% prediction interval that included the null value. These meta-analyses assessed the effect of ACEi on risk of hospitalisation, the effect of cardiovascular disease, chronic kidney disease, chronic lung disease and diabetes mellitus on risk of ICU admission, the effect of ACEi/angiotensin receptor blockers (ARBs) and sex on risk of acute kidney injury, the effect of body mass index on risk of invasive mechanical ventilation, the effect of chronic kidney disease and sex on the risk of a composite outcome (defined as ICU admission or death) and the effect of ARBs, insulin and low white blood cell count on risk of mortality. A forest plot of the 29 associations with $>1000$ events, $\mathrm{p}$-value $<0.005, \mathrm{I}^{2}<50 \%$ and no evidence of small-study effects is presented in figure 3. These 29 associations are visualised in a Sankey diagram (figure 4).

\section{Sensitivity analyses}

We repeated 57 meta-analyses after excluding the studies that reported only hazard ratios without information on the $2 \times 2$ contingency table (supplementary table S3). Overall, we observed high correlation of summary effect estimates $(\rho=0.98, p<0.05)$, p-values $(\rho=0.96, p<0.05)$ and $\mathrm{I}^{2}$ statistics $(\rho=0.95, p<0.05)$ before and after the exclusion of these studies.

192 meta-analyses were repeated after the exclusion of studies with < 100 COVID-19 participants (supplementary table S4). Overall, we observed high correlation of summary effect estimates $(\rho=0.99$, $\mathrm{p}<0.05)$, p-values $(\rho=0.96, \mathrm{p}<0.05)$ and $\mathrm{I}^{2}$ statistics $(\rho=0.96, \mathrm{p}<0.05)$ before and after the exclusion of these studies.

103 meta-analyses were repeated after the exclusion of studies including only COVID-19 patients with a specific comorbid disorder (supplementary table S5). Overall, we observed high correlation of summary effect estimates $(\rho=0.99$, $\mathrm{p}<0.05)$, p-values $(\rho=0.98, \mathrm{p}<0.05)$ and $\mathrm{I}^{2}$ statistics $(\rho=0.98, \mathrm{p}<0.05)$ before and after the exclusion of these studies. 


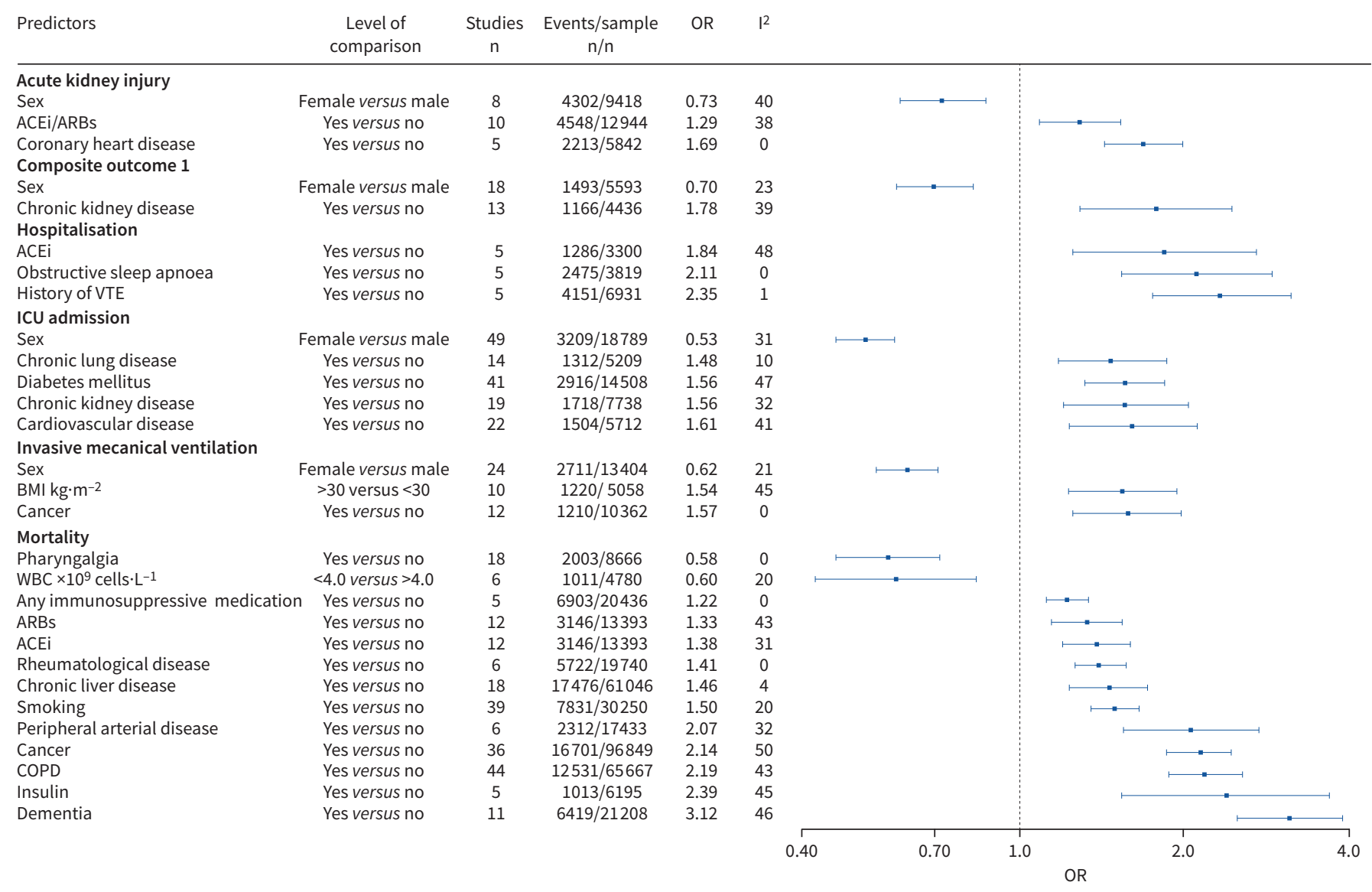

FIGURE 3 Forest plot of the 29 associations that had $>1000$ events, $p<0.005, \quad I^{2}<50 \%$ and absence of small-study effects. ACEi: angiotensin-converting enzyme inhibitor; ARB: angiotensin receptor blocker; VTE: venous thromboembolism; ICU: intensive care unit; BMI: body mass index; WBC: white blood cells.

\section{Discussion}

We conducted a comprehensive systematic review and meta-analysis to present an overview of the prognostic factors associated with any adverse outcome in patients diagnosed with COVID-19. We applied state-of-the-art approaches to combine the data from more than 420 studies by following the relevant methodological guidance. In our research effort, we considered more than 260 associations covering a wide range of predictors for multiple outcomes in COVID-19 patients. More than half of these associations presented a nominally significant effect, and only 16 of them provided strong evidence in terms of sample size, statistical significance, consistency and lack of small-study effects. We discuss the findings of our research effort with a focus on the most credible predictors, and the potential biases in these associations.

\section{Principal findings in context}

Our risk-of-bias assessment indicated that almost all the eligible studies of our systematic review presented high risk of bias in at least one domain of the QUIPS tool. Most articles had high risk of bias in the domains of participation and statistical analysis and presented several statistical pitfalls, including absence of a regression technique or inappropriate modelling strategy and handling of missing data, and poor reporting of the time horizon of prediction. In accordance with the systematic review of prognostic and diagnostic models for COVID-19 [24], our systematic review of individual prognostic factors also indicated poor reporting of the length of follow-up, exclusion of participants who had not experienced the event of interest by the end of the study period and inappropriate statistical methods. Our meta-analyses focused on unadjusted effect estimates, indicating that the prognostic effect of some prognostic factors is likely to become smaller in a multivariable prediction model [5]. We could not combine adjusted effect estimates, because they were scarcely reported in the literature. Whenever adjusted effect estimates were presented, selection of covariates was not consistent among different studies, whereas the selection of covariates in the multivariable model either was not clearly described in the eligible studies or was inappropriately 


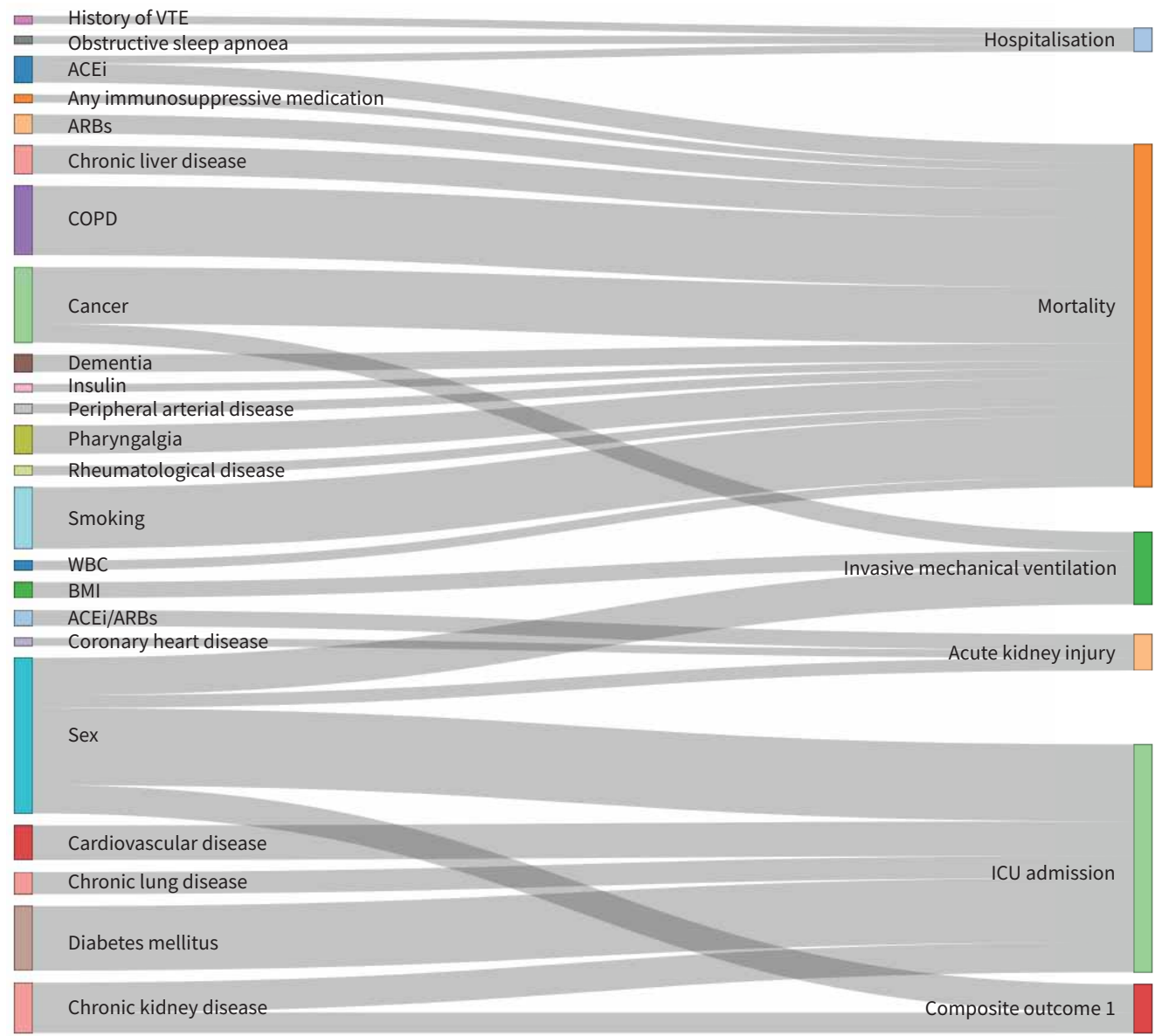

FIGURE 4 Sankey diagram presenting the 29 statistically significant associations at $p<0.005$ that had $>1000$ events, $I^{2}<50 \%$ and absence of small-study effects. The thickness of each line connecting a prognostic factor with an outcome depends on the number of studies examining this association. ACEi: angiotensin-converting enzyme inhibitor; ARB: angiotensin receptor blocker; VTE: venous thromboembolism; BMI: body mass index; WBC: white blood cells; ICU: intensive care unit.

based on the results of univariable modelling. An additional observation of our systematic review, corroborating the poor research practices in this field, was the substantial use of overlapping populations to examine the same or correlated prognostic factors and/or outcomes in different articles [25-27].

Age and sex constitute known prognostic factors for many chronic diseases. Our analysis confirms that age is a prognostic factor related to hospitalisation and mortality, and age presents a linear dose-response association with mortality. Although large between-study heterogeneity was observed in these meta-analyses, 95\% prediction intervals excluded the null value. In addition, sex was identified as a prognostic factor for ICU admission, acute kidney injury, invasive mechanical ventilation and a composite outcome (defined as ICU admission and death). In these associations small or moderate between-study heterogeneity was observed, but 95\% prediction intervals included the null value for acute kidney injury and composite outcome. The strong association of age and sex with adverse outcomes in COVID-19 patients indicates that they should be considered in multivariable prognostic models as covariates.

Symptoms and clinical signs associated with adverse events in COVID-19 patients could be used as "red flags" for patients requiring enhanced monitoring or treatment [28]. Dyspnoea was a predictor of hospitalisation; oxygen saturation was a predictor of mortality; and both associations presented large between-study heterogeneity with 95\% prediction intervals excluding the null value. Pharyngalgia was a predictor of hospitalisation with absence of large between-study heterogeneity and $95 \%$ prediction interval excluding the null value. Additionally, smoking could be considered a prognostic factor of clinical deterioration because available evidence showed a consistent association of smoking with risk of mortality. 
Almost half of the meta-analyses examined comorbid diseases as prognostic factors of COVID-19 outcomes. SARS-CoV-2 causes systemic inflammation and multi-organ damage outside the respiratory system, therefore patients with pre-existing chronic disorders of these organs may be more prone to organ insufficiency [29]. The most consistent evidence for a prognostic role were obstructive sleep apnoea, venous thromboembolism, cardiovascular disease, chronic kidney disease, chronic lung disease, diabetes mellitus, obesity, cancer, chronic liver disease, COPD, dementia, peripheral arterial disease and rheumatological disease, which predicted the occurrence of at least one prognostic outcome. An important limitation of the studies examining the prognostic effect of comorbid diseases was the vague reporting of their definition and ascertainment. This research practice could cause considerable heterogeneity, but we could not test this hypothesis in subgroup analyses. For example, presence of cardiovascular disease, chronic lung disease, chronic liver disease and any comorbidity constitute disease groups which might include different diseases across studies.

12 medications were considered as prognostic factors for COVID-19 patients. More than half of the relevant meta-analyses examined the prognostic significance of ACEi and ARBs. ACEi were identified as predictors of hospitalisation and mortality; ARBs were predictors of mortality; and the use of either ACEi or ARBs presented consistent evidence for an association with acute kidney injury. Among the rest of the medications, use of any immunosuppressive medication and insulin presented consistent evidence as predictors of mortality.

A total of 16 meta-analyses were performed for serum biomarkers and two adverse outcomes (i.e. mortality and ICU admission). Almost all these meta-analyses had a small sample. Only leukopenia presented consistent evidence for prediction of mortality, but the 95\% prediction interval included the null value. Although the majority of eligible studies dichotomised continuous biomarkers, many of them used standardised cutpoints. However, several studies did not report the cutpoint used, rendering this information ineligible for inclusion in meta-analysis. According to published guidance, we only combined studies that reported the cutpoint used, and used the same cutpoint, to avoid heterogeneity and to increase interpretability of results.

\section{Comparison with other studies}

Our findings on low study quality are in accordance with other systematic reviews that appraised published studies on COVID-19. A meta-epidemiological study that assessed all literature related to SARS-CoV-2 during the first phase of the pandemic showed that the majority of COVID-19 literature consists of articles without original data, whereas it assessed all the studies that contained original data and were published during that period as being at high risk of bias [6]. Moreover, a living systematic review and network meta-analysis of clinical treatment studies on COVID-19 patients and a recent systematic review of treatment studies of chloroquine and hydroxychloroquine also highlighted the low methodological quality of published studies [30, 31]. Furthermore, a systematic review of all prediction models (diagnostic and prognostic) for COVID-19 patients assessed all models as being at high risk of bias and highlighted the use of improper statistical analysis [24].

\section{Recommendations and policy implications}

There is a growing body of epidemiological studies examining prognostic factors of COVID-19. Due to the great significance of answering clinical questions related to prognosis of COVID-19, it is important to highlight the limitations and the gaps of the existing literature to improve the design and the validity of upcoming studies on COVID-19 prognosis. An important issue that should be raised is the need for adequate and transparent reporting of methodology and findings in future studies to improve the applicability of the evidence. The reporting of the methods and results of future studies could be improved by following the Strengthening the Reporting of Observational Studies in Epidemiology (STROBE) statement and the Prognosis Research Strategy (PROGRESS) framework [12, 32]. Future studies should include a detailed description of the recruitment process and sample selection, apply an appropriate regression modelling technique for the statistical analysis and present prognostic effects adjusted at least for age and sex. The time horizon for the prediction should be reported clearly and hazard ratios should be the effect size metric of choice for dichotomous outcomes, because odds ratios and risk ratios are sensitive to changes based on the time horizon of the prediction contributing to between-study heterogeneity. Furthermore, selective reporting of findings should be avoided to minimise the effect of publication bias in the reported associations.

Based on a comprehensive systematic review of COVID-19 related prediction models, it is more appropriate to base the selection of predictors for model building on previous literature than on a purely data-driven approach [24]. Considering that our meta-analyses synthesised unadjusted prognostic effect 
estimates, our findings do highlight the prognostic factors that could constitute reliable individual predictors for adverse events in patients with COVID-19. Our findings could be used as a pool for candidate predictors in future efforts to develop a new prediction model or update existing ones for COVID-19 patients.

\section{Strengths and limitations}

The major strength of our study is that it provides an overall mapping, and statistical synthesis of all published studies examining prognostic factors for multiple health outcomes in COVID-19 patients. We especially focused on including only studies with non-overlapping populations in each meta-analysis. This was achieved through detailed scrutiny of the methodology of eligible articles to capture the period of recruitment, and the hospital setting of each study. Furthermore, we enhanced our systematic review and meta-analysis by a risk-of-bias assessment using the QUIPS tool to identify the domains that could introduce bias to the prognostic factor's effect estimates.

However, our study has some limitations. We observed high risk of bias in at least one domain in almost all the studies, and this fact could lead to biased prognostic factor's effect estimates in the meta-analyses. Furthermore, half of the meta-analyses presented large between-study heterogeneity, but sources of heterogeneity could not be adequately explored due to poor reporting in the majority of articles. Potential sources of heterogeneity include different sampling methods and duration of follow-up, varying diagnostic criteria for COVID-19, inclusion of COVID-19 patients of various severity groups or stages of disease and use of different treatment regimens between studies. Incomplete reporting did not allow us to examine the effect of these characteristics on the summary effect of meta-analyses by subgroup analyses, sensitivity analyses or meta-regression. Moreover, a meta-analysis was not feasible for outcomes that were not defined in a standardised manner across different studies, such as progression of COVID-19, acute myocardial injury and acute liver injury.

\section{Conclusions}

Our article systematically identified and assessed all the studies examining prognostic factors for adverse outcomes in patients with COVID-19 published before the end of July 2020. We made an exhaustive and comprehensive effort to assess the quality of the studies and to exclude all possible duplicate studies and studies including overlapping population to avoid inflation of the reported summary effects. Our findings could facilitate the selection of candidate predictors for development or update of multivariable prognostic models.

Acknowledgement: We thank Richard D. Riley (Keele University, Keele, UK) for his constructive comments on an earlier version of the manuscript.

Data sharing: Additional data for the eligible studies are available on request from the corresponding author.

Author contributions: V. Bellou and L. Belbasis conceived and designed the study, and carried out the literature search, data extraction and statistical analyses. V. Bellou, L. Belbasis, I. Tzoulaki and E. Evangelou wrote the first draft. M. van Smeden and K.G.M. Moons critically commented on the first draft. All the authors wrote the final version of the manuscript. L. Belbasis accepts full responsibility for the work and conduct of the study, had access to the data, and controlled the decision to publish. The corresponding author attests that all listed authors meet authorship criteria and that no other meeting the criteria have been omitted. V. Bellou and L. Belbasis are the guarantors.

Conflict of interest: None declared.

Support statement: V. Bellou is supported by PhD scholarship funded by the Greek State Scholarships Foundation. No funding body has influenced data collection, analysis, or interpretation.

\section{References}

1 Zhu N, Zhang D, Wang W, et al. A novel coronavirus from patients with pneumonia in China, 2019. N Engl J Med 2020; 382: 727-733.

2 Sanders JM, Monogue ML, Jodlowski TZ, et al. Pharmacologic treatments for coronavirus disease 2019 (COVID-19). JAMA 2020; 323: 1824-1836.

3 World Health Organization. COVID-19 weekly epidemiological update, 16 February 2021. https://apps.who.int/ iris/handle/10665/339778 
$4 \quad$ Hingorani $A D$, van der Windt DA, Riley RD, et al. Prognosis research strategy (PROGRESS) 4: stratified medicine research. BMJ 2013; 346: e5793.

5 Riley RD, Moons KGM, Snell KIE, et al. A guide to systematic review and meta-analysis of prognostic factor studies. BMJ 2019; 364: k4597.

6 Raynaud M, Zhang H, Louis K, et al. COVID-19-related medical research: a meta-research and critical appraisal. BMC Med Res Methodol 2021; 21: 1.

7 Stroup DF, Berlin JA, Morton SC, et al. Meta-analysis of observational studies in epidemiology a proposal for reporting. JAMA 2000; 283: 2008-2012.

8 Cochrane Prognosis Methods Group. Tools. https://methods.cochrane.org/prognosis/tools Date last accessed: 1 June 2020.

9 Moons KGM, de Groot JAH, Bouwmeester W, et al. Critical appraisal and data extraction for systematic reviews of prediction modelling studies: the CHARMS checklist. PLoS Med 2014; 11: e1001744.

10 Chen Q, Allot A, Lu Z. Keep up with the latest coronavirus research. Nature 2020; 579: 193.

11 Chen Q, Allot A, Lu Z. LitCovid: an open database of COVID-19 literature. Nucleic Acids Res 2021; 49: D1534-D1540.

12 Riley RD, Hayden JA, Steyerberg EW, et al. Prognosis Research Strategy (PROGRESS) 2: prognostic factor research. PLoS Med 2013; 10: e1001380.

13 Moons KGM, Wolff RF, Riley RD, et al. PROBAST: a tool to assess risk of bias and applicability of prediction model studies: explanation and elaboration. Ann Intern Med 2019; 170: W1-W33.

14 Hayden JA, van der Windt DA, Cartwright JL, et al. Assessing bias in studies of prognostic factors. Ann Intern Med 2013; 158: 280-286.

15 Lawson R. Small sample confidence intervals for the odds ratio. Commun Stat Simul Comput 2004; 33: 1095-1113.

16 Higgins JPT, Thompson SG, Spiegelhalter DJ. A re-evaluation of random-effects meta-analysis. J $R$ Stat Soc Ser A Stat Soc 2009; 172: 137-159.

17 DerSimonian R, Laird N. Meta-analysis in clinical trials. Control Clin Trials 1986; 7: 177-188.

18 Higgins JPT. Commentary: heterogeneity in meta-analysis should be expected and appropriately quantified. Int J Epidemiol 2008; 37: 1158-1160.

19 Higgins JPT, Thompson SG. Quantifying heterogeneity in a meta-analysis. Stat Med 2002; 21: 1539-1558.

20 Egger M, Davey Smith G, Schneider M, et al. Bias in meta-analysis detected by a simple, graphical test. BMJ 1997; 315: 629-634.

21 Benjamin DJ, Berger JO, Johannesson M, et al. Redefine statistical significance. Nat Hum Behav 2018; 2: 6-10.

22 Bellou V, Belbasis L, Konstantinidis AK, et al. Elucidating the risk factors for chronic obstructive pulmonary disease: an umbrella review of meta-analyses. Int J Tuberc Lung Dis 2019; 23: 58-66.

23 Belbasis L, Dosis V, Evangelou E. Elucidating the environmental risk factors for rheumatic diseases: an umbrella review of meta-analyses. Int J Rheum Dis 2018; 21: 1514-1524.

24 Wynants L, Van Calster B, Collins GS, et al. Prediction models for diagnosis and prognosis of Covid-19 infection: systematic review and critical appraisal. BMJ 2020; 369: m1328.

25 von Elm E, Poglia G, Walder B, et al. Different patterns of duplicate publication: an analysis of articles used in systematic reviews. JAMA 2004; 291: 974-980.

26 Broad W. The publishing game: getting more for less. Science 1981; 211: 1137-1139.

27 Glasziou P, Sanders S, Hoffman T. Waste in Covid-19 research. BMJ 2020; 369: m1847.

28 Hemingway $\mathrm{H}$, Croft $\mathrm{P}$, Perel $\mathrm{P}$, et al. Prognosis research strategy (PROGRESS) 1: a framework for researching clinical outcomes. BMJ 2013; 346: e5595.

29 Jose RJ, Manuel A. COVID-19 cytokine storm: the interplay between inflammation and coagulation. Lancet Respir Med 2020; 8: e46-e47.

30 Alexander PE, Debono VB, Mammen MJ, et al. COVID-19 coronavirus research has overall low methodological quality thus far: case in point for chloroquine/hydroxychloroquine. J Clin Epidemiol 2020; 123: 120-126.

31 Siemieniuk RA, Bartoszko JJ, Ge L, et al. Drug treatments for Covid-19: living systematic review and network meta-analysis. BMJ 2020; 370: m2980.

32 von Elm E, Altman DG, Egger M, et al. The Strengthening the Reporting of Observational Studies in Epidemiology (STROBE) statement: guidelines for reporting observational studies. J Clin Epidemiol 2008; 61: 344-349. 